\title{
An Interpretative Phenomenological Analysis of Families Affected by Autism in Dubai
}

\author{
Tania Sopaul \\ Department of Psychology, Manchester Metropolitan University, Manchester, UK
}

\section{Keywords}

Autism spectrum disorder · Dubai population · Clinical psychology · Educational psychology · Evidence-based

\begin{abstract}
Background: The prevalence of autism spectrum disorders (ASD) in the Middle East region is suggested to be comparable with rates reported in the Western World; however, in the United Arab Emirates (UAE), autism is under-diagnosed and over-looked. Objectives: This qualitative study based in Dubai, $U A E$, aimed to explore personal experiences of autism and investigate the decision-making process of parents. A focus group interview with 6 UAE national mothers who have an autistic child was conducted; the semi-structured interview was audio-recorded and transcribed. Method: Interpretative phenomenological analysis was performed on the interview transcript and 3 main themes emerged: Education, Support and Social Stigma. Results: The research concluded that the majority of mothers are primary caregivers and the challenges faced by autistic families include: a lack of understanding and research; family reputation; stigma-associated discrimination; and maternal stress. Conclusions: Mothers with autistic children experience a number of stressors and would benefit from educational workshops, including ASD-
\end{abstract}

specific training. A call for further investigation into the role and impact of maids as primary caregivers for autistic children in the UAE is advocated.

(C) 2019 The Author(s)

Published by S. Karger AG, Basel

\section{Introduction}

Mental health is an emerging field in the Arab world; much of the research conducted has focused on the effectiveness of special educational needs and teaching interventions, whilst there has been limited research which investigates the impact of disability on individuals and family members [1, 2]. Abbady et al. [3] reported prevalence estimates of 199 children with autism spectrum disorders (ASD) in Dubai of which 29.1\% were Emirati nationals. A surprising figure when local Emiratis make up only $10 \%$ of the population with the remainder being considered expatriates [4]. Although the aetiology of ASD is not fully understood, consensus for an epigenetic process involving environmental and genetic interactions exists [5].

The need for pathways that allow people with disability to access services effectively is advocated [6]. In an attempt to encourage reform, "Law No. 2 of 2014 Concern- 
ing Protection of the Rights of Persons with Disabilities in the Emirate of Dubai" has been passed [7]. This law aims to provide high-quality provision for disabled people, including government-subsidised education and healthcare, as well as encouraging public awareness and social integration. However, the implementation of provisions must also be contextualised to this part of the world, where reform is still emerging as a slow evolutionary process.

Common [8] proposed a number of systems that resist international reform trends, for example, centralised political systems and strong cultural, traditional habits, demonstrating the preference for domestic, as opposed to international, guidelines. There is also scepticism as to whether government agendas to recruit disabled people in to the work force are being fulfilled, with the transition from school to work a real grey area [9].

Accumulating evidence suggests that the profile of current research in the United Arab Emirates (UAE) needs to be raised to help bridge the gap between the needs of and services for autism [10]. Additional research advocates the need for sample populations from all 7 emirates of the UAE. Kelly et al. [11] examined the treatment of autism in Abu Dhabi where speech and language therapy was the most common treatment, followed by technology-based treatments and applied behaviour analysis. To address the apparent gap in literature from all emirates of the UAE, the current research aimed to fill this void. The current study is also in line with Kelly et al. [11] recommendation for future research to use the qualitative analysis method to explore personal experiences of autism and investigate the decision-making process of parents.

The apparent under-diagnosis of autism sees the occurrence of autistic characteristics associated with other factors like male gender, behavioural issues and a family history of developmental delay [12]. A lack of recognition for conditions like autism is attributed to cultural factors where parents do not want to "expose" their child to the world [13]. It is claimed that in the Emirate state of Oman, traditional views that are fixated on family reputation can cause families to neglect their disabled child and even hide their existence. The implications of this mindset are profound, as there could be a significant percentage of children still unaccounted for.

Khamis [14] proposed that, despite the effort put into provision, it is feelings of shame, stigma, guilt and denial that see disabled children's access to early intervention delayed. To verify this notion, Abbady et al. [3] concluded that families are not going to consult general practitio-

IPA of Families Affected by Autism in Dubai ners for early diagnosis of ASD for cultural reasons. An alternative possibility is parents are left feeling alienated after seeking clinical advice and thus go on to pursue traditional therapies instead [13]. Research which engages the parents of ASD children is required, in accordance with this the current study will recruit Emirati national mothers.

Although little is known about the direct impact of consanguinity on the development of autism, it is important to note that Arab countries hold the highest rates for consanguineous marriage worldwide, specifically $30 \%$ are accounted for by first cousin marriages [15]. Related to this notion, research highlights the issue of high consanguinity rates in Arab families associated with a higher incidence of congenital abnormalities and intellectual disability [16]. This issue is particularly pertinent in many parts of the Arab world where society is still tribal, making it difficult to assess the epidemiology of genetic disease, which is often localised to specific villages, communities, families and tribal groups [15].

Raising a child diagnosed with a behavioural disorder is suggested to significantly impact on mothers, who are considered primary caregivers [17]. Khoadee and Koolaee [18] found, when comparing ASD mothers to nonASD mothers, that $66 \%$ felt excluded and that their social lives were non-existent. Despite fathers being assigned traditional patriarchal roles, there is a transforming role for mothers as primary caregivers in the UAE; a transition from stay-at-home mothers to career women who rely on maids to raise their children exists $[19,20]$. The reliance on home help in UAE culture has been criticised by Roumani [21] who claimed that only $8 \%$ of maids were specifically hired and trained to look after children. The remainder reported that caring for their employer's child was an additional household chore.

Extensive evidence implicates a critical role for maids on child development that promotes learned helplessness and familial attachment, where the maid assumes maternal surrogacy [20,22]. Maids are often illiterate in Arabic language with little education and training; this manifests as a lack of stability, poor behaviour and linguistic confusion, which could lead to difficulties in language acquisition amongst ASD children [23, 24]. An adequately trained and stable childcare provider to promote the positive social and emotional development of ASD children is advocated [25]. The impact of hiring maids to raise autistic children is a complex and understudied field; consequently, the exploration of family experiences in the current study could provide insight to illuminate this phenomena. 
Fig. 1. A thematic map of emerging themes

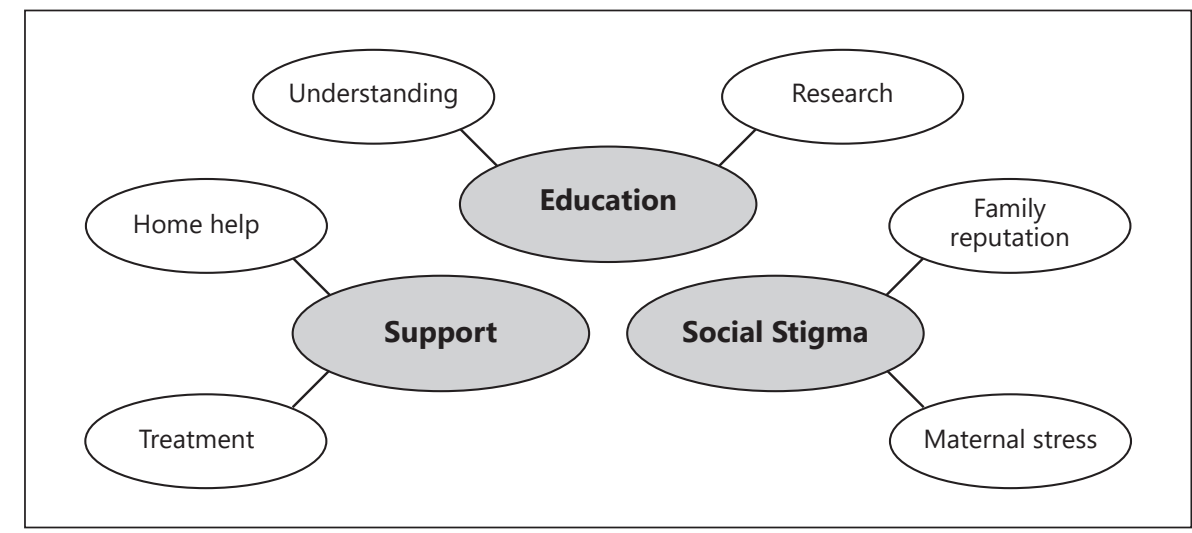
and subthemes.

\section{Aim}

There is a call for research to address the issue of public understanding and to raise the awareness of special needs and disability in the UAE [11-13]. The current research will aim to explore the major challenges faced by autistic children and their families in Dubai with the following proposed research questions:

To explore the role of the family in parenting a child with autism.

To gain an insight in to the current provisions and mental health awareness in the UAE.

To identify the major challenges faced by families with autistic children in the UAE.

\section{Methodology}

\section{Sample}

The qualitative approach enables high-quality, insightful data to be collected [26] whilst the participants' emotions and experiences are encapsulated and the partiality of the researcher is accepted [27]. Participants in this research study belonged to a similar social identity: 6 females, aged between 40 and 50 years, Emirati nationals and mothers of autistic children. This is line with research that advocates for a small homogenous sample for interpretative phenomenological analysis (IPA) $[28,29]$. Participants in the current study were recruited through volunteer sampling; an advertisement was issued to the parents of autistic children at a local special needs clinic [30].

\section{Procedure}

A semi-structured interview using open-ended questions was implemented; this method is suggested to facilitate group exploration of depth required to identify the similarities and differences between participants [31, 32]. The semi-structured interview held at a local educational institute in Dubai, followed by a schedule for guided discussion (where necessary) and was audio recorded then transcribed. IPA was conducted; this protocol involved reading the interview transcript, taking descriptive notes in page margins, identification of over-arching themes and subthemes with supporting quotations and creation of a thematic map. A theme is defined as dialogue that encapsulates meaning in relation to the research question; the proposed procedure enables access to nuances and validation of data $[26,28,29,33]$.

\section{Results}

IPA was conducted following the protocol suggested by Smith and Osborn [28]. As illustrated by Figure 1 the main themes which emerged from this research are Education, Support and Social Stigma. These were further subdivided into the subthemes of understanding, research, home help, treatment, family reputation and maternal stress. Excerpts from participants were extracted from the interview transcript where appropriate and compared with published literature.

\section{Discussion}

\section{Education: Understanding}

One of the most poignant superordinate themes expressed by all of the mothers was a lack of understanding and confusion as to how best they can support their autistic children. Sarah discusses the barriers she faces when trying to access information,

"Before coming to this clinic, it was very difficult to find information about autism... The only available information is online chat rooms, you don't know if this is always correct" (Sarah, P3, L 111-113). 
There are many indicators in Sarah's account that exemplify her struggle to gain access to credible information about ASD. She is sceptical about the use of online forums and questions whether the information available is trustworthy; this suggests Sarah is in doubt. Samadi and McConkey [34] have shown that the parents of autistic children in the Arab world indicated speaking to other parents, as their most popular method of accessing information, whilst only $12 \%$ mentioned using the internet. This is in stark contrast to the Western world where $86 \%$ of parents in the US indicated using websites [34]. The differences in internet usage observed by Samadi and McConkey [34] could be a result of reservations held by family members, as with Sarah's case. Alternatively, there are other factors to consider like IT literacy and strict censorship of online material, which is not in line with UAE law. A reliance on other parents for information can encourage inaccurate and misinformed judgements of ASD.

To address this issue, indigenous education courses facilitated by trained parents of autistic children have been suggested. Barbera [35] found that trained parents were able to build a positive rapport and transfer correct ASD information to other parents.

Aisha makes a desperate appeal to try and get answers to the questions she does not understand,

"We just...sometimes, do not understand why has this happened? What has caused this? What have we done wrong? Nobody has shown us how to support him, there is no rule book, you know..." (Aisha, P11, L 510-512).

Aisha's upset and frustration is evident; she portrays a sense of, loss of control and similarly with Sarah implies that an obstacle she faces is gaining access to information. The implications of this lack of understanding could affect the diagnosis of autistic children. Eapen et al. [12] highlighted a lack of understanding, education and awareness resulting in apparent under-diagnosis of ASD in the UAE.

Tara highlights the issue of social and cultural views impeding on clinical diagnosis.

"The doctors, they tell us there is nothing wrong with your child..." (Tara, P11, L 541).

The existence of mental health issues is often underplayed in the UAE; instead, social and cultural factors are blamed. Al-Farsi et al. [36] have found that Arab society will often hold parents accountable for their child's autism, signifying that the child is spoilt. In a similar vein,
Ghuloum et al. [37] identified religion and superstition as playing a significant role. This notion is supported by AlAdawi et al. [38] who concluded that Arab society favoured superstition over genetic aetiological factors for ASD. Other beliefs include the violation of a religious code has caused a child with a disability to be born [10]. In all instances, a child with autism is usually perceived as a burden to the family, for whom they must accommodate.

\section{Education: Research}

ASD research in the Arab world is perceived to be very much in its infancy; this notion is reflected in Lisa's commentary,

"My son was included in a research project, they observed his behaviours... but we have had no further information, we do not know what information was found, the researcher has not been back to the clinic" (Lisa, P11, L 516-518).

Lisa has suggested that there are communication difficulties with the current ASD research agenda, in line with Hussein et al. [10] who claimed that research on the provisions for ASD in the UAE needs to be raised.

The following extract highlights the issue of utilising evidence-based practice to inform healthcare programmes. Tara discloses her experiences of a family history of autism,

"Really you don't know any other families?... I know very many, my sister has an autistic son as well and my cousin, he too has an autistic child, there are lots of Emirati families who have the same problem" (Tara, P5, L 267-270).

Tara's family history of ASD could be explained by the suggested link between consanguineous marriage and a heightened risk of autism prevalence [39]. The results of this genetic analysis showed that Middle Eastern families, where inbreeding was prevalent, had an average of $2 \mathrm{au}-$ tistic children. Hence, the apparent occurrence of autism in Emirati families could be a reflection of the local culture and traditions where intermarriage between closely related bloodlines has been apparent for generations [15]. Our findings are in agreement with previous work demonstrating a need for research on consanguineous marriage in societies with high rates of autism in order to provide a comprehensive genetic service as part of the preventative healthcare programme [40].

\section{Support: Home Help}

The mothers feel that mainstream education systems have failed their autistic children and family. 
"Mashallah there has been some advance, when she was a child no school would take her, but now there are special schools for our children" (Mina, P2, L 52-53).

Mina is complimentary about the improvements that have been made to the provisions for autistic children. She verbalises a sense of gratitude and acceptance for both her autistic child and her family as a whole. Mina's commentary of inclusive educational practice is reflective of the UAE inclusive schools initiative held by the Ministry of Education (2015). Local Emirate nationals receive a free public education and it is a requirement for mainstream schools to provide the support and provisions required for special educational needs children. However, despite the advances, there still remains inconsistency, the fate of students after they leave mainstream education remains unclear, autistic children do not seek employment nor do they go to study at university. The implications of this lack of provision post 16 would suggest that they remain in a state of limbo, completely reliant on their families to support and care for them. A need for an adult autism centre specialising in the care of adolescence after the age of 18 , once they have left the safe and sheltered environment provided by schools, is advocated [3].

Sarah gives an emotive account of the barriers she has faced when trying to get her autistic son, Zach in to a mainstream school,

"The teachers said they did not have the required training to deal with Zach's behavioural problems, he was even put into the special class to help him to focus and get one to one support but this did not help" (Sarah, P3, L 121-123).

Dissimilar to Mina, Sarah expresses her contempt at the mainstream schooling system. She feels particularly let down by the teachers and implies that they did not possess the training required to deal with Zach's challenging behaviour. The teachers' perception of disability is an important factor to consider here. Mereoiu et al. [25] claim full inclusion of young children with ASD remains challenging; some of the barriers faced by educators include insufficient planning and instructional time; a lack of training for early childhood teachers and inadequate resourcing.

Sarah later went on to speak of her dependence on home help to support the learning needs of her autistic child,

\footnotetext{
"Zach has a maid who stays with us, she is not a qualified teacher but speaks English fluently and mashallah is very patience with Zach" (Sarah, P11, L 529-530).
}

Feelings of relief are conveyed as Sarah praises her maid's patience when caring for Zach, she considers this a time of escapism whereby she can detach as the primary caregiver. The reliance on home help in UAE culture has been criticised as young, inexperienced women with 70 contact hours per week, double the recommended guideline, are exploited [21]. It has been suggested that a dependence on maids can result in cases, where the maid is the primary caregiver and is not equipped with the skills or emotional attachment required to raise the child; maids are often hired on the premise of homecare and eventually forcibly drawn to childcare [24].

\section{Support: Treatment}

The mothers seek remedial treatments for their autistic children and appear to be more concerned with curing the symptoms, rather than appreciating the underlying cause. Aisha, in particular, is very adamant to learn a "cure" for her son,

"I want to know, what is the treatment for this condition? How can we make Ahmed normal, like the other children? (Aisha, P11, L 521-522).

"Normal" implies that Aisha perceives Ahmed in a negative light and that she is taking ownership for his ASD. This is in line with previous findings that show that $16.4 \%$ of Dubai families studied were actively seeking help for ASD assessment in 6- to 18-year-old children; such research also indicates late-stage diagnosis, effective school health strategies and training of GP's to aid early diagnosis of ASD [3]. Evidently, these studies would suggest that ASD research is being conducted in Dubai; however, the extent to which this research is utilised to inform evidence-based practice remains unclear. Kelly et al. [11] examined the treatment of autism in the UAE; the findings in this research study showed a lack of consistency in the licensing and federal policy for treatments like applied behaviour analysis and an overall lack of evidence to support the efficacy of chosen treatments.

An absence of regulation for autism intervention in the UAE sees some parents turning to alternative, traditional medicine.

\footnotetext{
"Yes this is my question, what is a suitable treatment? There are no medications provided by the Doctors instead we take Dawud to get traditional remedies.... like Al-Hijama” (Tara, P12, L 545-546).

The use of culture-specific intervention for the treatment of autism in Arabian nations is supported by Kelly et al. [41] who have identified the use of alternative medicine including Al-Hijama, acupuncture and vitamin supplements. Al-Hijama or wet cupping is a form of alternative medicine that alleviates pain and detoxifies the body,
}

86

Dubai Med J 2019;2:82-89

DOI: $10.1159 / 000501770$
Sopaul 
a cultural practice in the Arab world. The use of vitamin and mineral supplements to treat autistic symptoms is supported by Adams et al. [42] who have observed significant improvements in hyperactivity and tantrums in autistic children. The vitamin and mineral supplements are believed to be pre-cursors for the production of neurotransmitters, therefore improving the nutritional and metabolic pathways that affect neuronal functioning [42].

\section{Social Stigma: Family Reputation}

There is an apparent underlying issue of reputation that the mothers and their families feel threatened by.

"People like to talk... when your child is not the same... there is more focus and the talk is about the mother and how she raises her children" (Mina, P3, L 141-142).

Mina opens up about her experience of being in the public eye. She implies that it is the mother of the autistic child who is often blamed, stereotyped and excluded. Kinnear et al. [43] concluded that $95 \%$ of parents felt that their autistic children were stigmatised. The child's autism-related behaviours are believed to impact on the social processes of stereotyping, rejection and exclusion.

Courtesy stigma is experienced by the parents and family members of the disabled child; associated variables have been highlighted which include blaming family members for the deterioration of the child's condition and holding the attitude that others could be contaminated and should be pitied [44]. In this instance, it is proposed that the blaming of caregivers for the onset of autism results in parents who find it challenging to embrace a connection, instead detach themselves from their diagnosed child [45].

The perception that an autistic child's clinical diagnosis reduces them from a normal whole person to a spoiled individual is captured in Mina's statement,

"Now who will carry on the family name?" (Mina, P12, L 550).

Mina's question encapsulates the extreme social and cultural pressure imposed on the families as they are fixated with their reputation. Her statement suggests that her autistic son is not worthy of carrying the family name, which has enforced strain on family relationships. Profanter [13] highlighted the importance placed on reputation; it is suggested that in the Emirate state of Oman, the stigma associated with having a disabled child results in families hiding their identity from the public. This often lends itself to the child being housebound, without access to education and remedial provisions.

IPA of Families Affected by Autism in Dubai

\section{Social Stigma: Maternal Stress}

Traditional gender roles are prevalent; mothers are primary caregivers whilst fathers work. This is demonstrated in Sarah's commentary,

"My husband is away working a lot of the time, I am left with Zach... it is hard you know... I have 2 younger children to care for and Zach" (Sarah, P3, L 170-172).

In support of this notion, Benson [17] claimed that when a child is diagnosed with a behavioural disorder, mothers significantly suffer. The mothers are shown to be more affected when a female is diagnosed with ASD. This notion is expressed in a dialogue with Lisa,

"I am very worried for the future of my daughter, who will she marry? Who will look after her when I am gone" (Lisa, P6, L 334335).

In contrast to this Jaya provides an alternative perspective, she disagrees and suggests that the challenging behaviours of autistic boys are far more difficult to manage than girls,

“... but I think, for boys it is more difficult to control the bad behaviours, my son, he is always screaming, shouting, irritable, very hyperactive and I find it hard to communicate with him" (Jaya, P6, L 340-343).

\section{She then goes on to say,}

"My friend has a daughter who is autistic, yes she sometimes screams but she is very calmer compared to Jamal" (Jaya, P6, L 347).

The comments made suggest that autism is perceived and treated differently for boys and girls. There is an apparent gender division, the mothers believe that females with autism are easier to care for and are calmer, whilst males are more aggressive and violent. Trubanova et al. [46] highlighted gender inequality in the diagnosis and treatment of ASD, with a reported 5:1 male to female ratio. They suggest that females are faced with issues of diagnostic overshadowing, whereby a clinical diagnosis of autism is instead often associated with depression or anxiety. The socialisation of females is also suggested to impact the gender division; a stereotypical personality trait is more likely to see women perceived as shy or socially withdrawn. Therefore, this could explain the disparity observed by the mothers; the religious and cultural context of the UAE sees women as submissive to men. Young women especially are often obedient, shy and conforming, which is perhaps why autistic girls are perceived as easier to manage than autistic boys. 


\section{Conclusion}

This research study can establish that mothers with autistic children in Dubai experience a number of stressors and would benefit from educational workshops, including ASD-specific training. Although there have been some advances in recent times, with the publication of research increasing [41], there remains an issue of evidence-based practice that is an emerging field in the UAE. There is a need for research findings from consanguineous marriage studies to be applied to genetic screening for preventative healthcare programmes [15]. The accounts showed that parents struggle to find relevant and accurate information to inform their own evidence-based practice. The use of trained parents of ASD children, to deliver programmes to other parents, has been shown to be an effective method to overcome this problem [35]. A recommendation is for future research to study the role and impact of maids as primary caregivers for autistic children in the UAE.

Although research advocates a small homogenous sample for IPA, there are limitations to this approach [28, 29]. In this research, an ethnocentric and gynocentric sample of Emirati national mothers restricts the generalisation of research findings to men in the Western World. Volunteer sampling is subject to bias as a highly motivated group of individuals is selected; this research also employed a small sample size, a recommendation for future research is to increase the sample population. Disclosure of information was restricted by social and cultural factors involving privacy and reputation. Participants showed varying levels of engagement during the semi-structured interview; some made limited contribution and were overshadowed by members of the focus group. Verbatim extracts were included for IPA, an illuminating process that helps the reader to trace the analytic process; however, greater acknowledgement of the analysts' preconceptions and reflexivity is advocated to increase transparency and enhance rhetorical power [47].

\section{Acknowledgements}

I would like to thank the participants for partaking in this research study. I sincerely thank Jeremy Hopper academic tutor at Manchester Metropolitan University for his supervision in carrying out this research study.

\section{Statement of Ethics}

The research study received ethical approval from the Manchester Metropolitan University Psychology Department and conformed to the British Psychological Society ethical guidelines. A number of procedures were carried out in line with ethical practice; these include the completion of an ethics approval form and gaining participants' informed consent.

Throughout the research study, all participants were informed of their right to withdraw and debrief was delivered following the interview. The participant's names were anonymised and pseudonyms were chosen by the researcher, for the completion of data analysis and the research report. Data was audio recorded and stored using an encrypted file; once transcribed, audio files were deleted within a 2-week duration from the interview date. The research study was designed to avoid harm or untoward experiences for participants.

\section{Disclosure Statement}

The authors have no conflicts of interest to declare.

\section{Funding Sources}

The authors have no funding sources to declare.

\section{Author Contribution}

T.S. first-author conceived and designed the research; collected the data; performed the analysis; wrote the paper and prepared critical revisions for final publication. T.S conducted the research whilst residing in Dubai and studying a distance learning MSc Psychology course at Manchester Metropolitan University accredited by the British Psychological Society. T.S. (2019) An IPA of families affected by Autism in Dubai.

\section{References}

1 El-Sweidi M, Abu Shehab A. Survey of disabilities in the United Arab Emirates families. UAE: Ministry of Labour and Social Welfare; 1995.

2 Dukmak S, Alghazo I. The teaching effectiveness of special education teachers in the United Arab Emirates. UAE: Fifth UAE university research conference; 2004.
3 Al-Abbady K, Hessian HY, Alaam MW. Prevalence, Trend, Determinants and Prediction of Autism Spectrum Disorders among Dubai Population, Diagnostic and Management Contexts. Ped Health Res. 2017;2(2):2.
4 Dubai Statistics Centre. (2015) Population Bulletin Emirate of Dubai 2015. [Online] [Accessed 18th July 2017]. Available from: https://www.dsc.gov.ae/Publication/Population $\% 20$ Bulletin $\% 20$ Emirate $\% 20$ of $\% 20$ Dubai\%202015.pdf. 
5 Habib SS, Al-Regaiey K, Bashir S, Iqbal M. Role of Endocannabinoids on Neuroinflammation in Autism Spectrum Disorder Prevention. J Clin Diagn Res. 2017 Jun;11(6):CE0103.

6 Hamdy H, Anderson MB. The Arabian Gulf University College of Medicine and Medical Sciences: a successful model of a multinational medical school. Acad Med. 2006 Dec;81(12): 1085-90.

7 International Labour Organisation. (2015) Law No. 2 of 2014 Concerning Protection of the Rights of Persons with Disabilities in the Emirate of Dubai 2014-2015. [Online] [Accessed 18th July 2017]. Available from: http:// www.ilo.org/dyn/natlex/natlex4.detail? p $_{-}$ lang $=\& p \_i s n=98967 \& p \_$classification $=08.01$.

8 Common R. Administrative change in the Gulf: modernization in Bahrain and Oman. Int Rev Adm Sci. 2008;74(2):177-93.

9 Dev S, Kumar J. Teacher's Perception towards Integration of Learning Disabled Students into Regular Class Room - A Study in Dubai \& Abu Dhabi Schools. Procedia Soc Behav Sci. 2015;211(1):605-11.

10 Hussein H, Taha G. Autism spectrum disorders: A review of literature in the Arab countries. MECPsych. 2013;20(1):106-16.

11 Kelly MP, Alireza I, Busch HE, Northrop S, Al-Attrash M, Ainsleigh S, et al. An overview of autism and applied behavior analysis in the Gulf Cooperation Council in the Middle East. Rev J Autism Dev Disord. 2016;3(2):154-64.

12 Eapen V, Mabrouk AA, Zoubeidi T, Yunis F. Prevalence of pervasive developmental disorders in preschool children in the UAE. J Trop Pediatr. 2007 Jun;53(3):202-5.

13 Profanter A. Facing the challenges of children and youth with special abilities and needs on the fringes of Omani society. Child Youth Serv Rev. 2009;31(1):8-15.

14 Khamis V. Psychological distress among parents of children with mental retardation in the United Arab Emirates. Soc Sci Med. 2007 Feb; 64(4):850-7.

15 Tadmouri GO, Nair P, Obeid T, Al Ali MT, Al Khaja N, Hamamy HA. Consanguinity and reproductive health among Arabs. Reprod Health. 2009 Oct;6(17):17.

16 Abdulrazzaq YM, Bener A, al-Gazali LI, alKhayat AI, Micallef R, Gaber T. A study of possible deleterious effects of consanguinity. Clin Genet. 1997 Mar;51(3):167-73.

17 Benson PR. Network characteristics, perceived social support, and psychological adjustment in mothers of children with autism spectrum disorder. J Autism Dev Disord. 2012 Dec;42(12):2597-610.

18 Khodaee MR, Koolaee AK. Predictors of metal health distress in mothers with autism children. Eur Psychiatry. 2011;26(1):312-8.
19 Al-Najjar S. Women Migrant Domestic Workers in Bahrain (International Migration Papers). Geneva: International Labour Office; 2002.

20 Hayden M. Introduction to International Education: International Schools and their Communities. London: Sage Publications; 2006.

21 Roumani HB. Maids in Arabia the impact of maids as carers on children's social and emotional development. J Early Child Res. 2005; 3(2):149-67.

22 Parrenas R. Servants of Globalization: Women, Migration and Domestic Work. Stanford (CA): Stanford University Press; 2001.

23 Biggs $M$. The United Arab Emirates and its Education: Selected Themes and Issues with Reference to the 'Small Country' Context. Ph.D. The University of Hull; 1995.

24 Khalifa B. 'Parental treatment of children and self-efficacy and the relation with the domestic servant.' AlAzhar University-. Educ J. 2009; 139:553-94.

25 Mereoiu BM, Dobbins C, Niemeyer JA. Exploring perspectives on child care with families of children with autism. Early Child Res Pract. 2015;17(1):13.

26 Braun V, Clarke V. Successful Qualitative Research: a practical guide for beginners. London: Sage; 2013.

27 Willig C. Introducing Qualitative Research. 3rd ed. Berkshire: Open University Press; 2013.

28 Smith JA, Osborn M. Interpretative phenomenological analysis. In: Smith JA, editor. Qualitative psychology: A practical guide to research methods. London: Sage; 2003. pp. 53-80.

29 Bloor M, Frankland J, Thomas M, Robson K. Focus groups in social research. London: SAGE Publications; 2001. https://doi. org/10.4135/9781849209175.

30 Bernard HR. Research methods in anthropology: Qualitative and quantitative approaches. 3rd ed. Walnut Creek (CA): Alta Mira Press; 2002.

31 Willig C. Introducing qualitative research in psychology: adventures in theory and method. New York (NY): Open University Press; 2006.

32 Smith JA, Flowers P, Larkin M. Interpretative Phenomenological Analysis: Theory, research and methods. London: Sage; 2009.

33 Barriball KL, While A. Collecting data using a semi-structured interview: a discussion paper. J Adv Nurs. 1994 Feb;19(2):328-35.

34 Samadi SA, McConkey R. Autism in developing countries: lessons from iran. Autism Res Treat. 2011;2011(2):145359.

35 Barbera ML. The experiences of Autism Mothers who become behaviour analysts: A qualitative study. J Speech Lang Pathol Appl Behav Anal. 2007;2(3):287-305.
36 Al-Farsi YM, Al-Sharbati MM, Al-Farsi OA, Al-Shafaee MS, Brooks DR, Waly MI. Brief report: prevalence of autistic spectrum disorders in the Sultanate of Oman. J Autism Dev Disord. 2011 Jun;41(6):821-5.

37 Ghuloum S, Bener A, Abou-Saleh MT. Prevalence of mental disorders in adult population attending primary health care setting in Qatari population. J Pak Med Assoc. 2011 Mar; 61(3):216-21.

38 Al-Adawi S, Dorvlo AS, Al-Ismaily SS, AlGhafry DA, Al-Noobi BZ, Al-Salmi A, et al. Perception of and attitude towards mental illness in Oman. Int J Soc Psychiatry. 2002 Dec; 48(4):305-17.

39 Morrow EM, Yoo SY, Flavell SW, Kim TK, Lin Y, Hill RS, et al. Identifying autism loci and genes by tracing recent shared ancestry. Science. 2008 Jul;321(5886):218-23.

40 al-Gazali LI, Dawodu AH, Sabarinathan K, Varghese M. The profile of major congenital abnormalities in the United Arab Emirates (UAE) population. J Med Genet. 1995 Jan; 32(1):7-13.

41 Kelly MP, Tennant L, Al-Hassan S. Autism treatments used by parents in Abu Dhabi, United Arab Emirates. Poster session presented at 41st Annual Convention of the Association for Behavior Analysis International, San Antonio, 2015.

42 Adams JB, Audhya T, McDonough-Means S, Rubin RA, Quig D, Geis E, et al. Effect of a vitamin/mineral supplement on children and adults with autism. BMC Pediatr. 2011 Dec; 11(11):111.

43 Kinnear SH, Link BG, Ballan MS, Fischbach RL. Understanding the Experience of Stigma for Parents of Children with Autism Spectrum Disorder and the Role Stigma Plays in Families' Lives. J Autism Dev Disord. 2016 Mar;46(3):942-53.

44 Milačić-Vidojević I, Gligorović M, Dragojević $\mathrm{N}$. Tendency towards stigmatization of families of a person with autistic spectrum disorders. Int J Soc Psychiatry. 2014 Feb;60(1):6370.

45 Gray DE. Everybody just freezes. Everybody is just embarrassed: felt and enacted stigma among parents of children with high functioning autism. Sociol Health Illn. 2002;24(6): 734-49.

46 Trubanova A, Donlon K, Kreiser NK, Ollendick TH, White SW. Underidentification of Autism Spectrum Disorder in Females: A Case Series Illustrating the Unique Presentation of this Disorder in Young Women. Scand J Child Adolesc Psychiatr Psychol. 2014;2(2): 66-76.

47 Brocki JM, Wearden JA. A critical evaluation of the use of interpretative phenomenological analysis (IPA) in Health Psychology. Psychol Health. 2006;21(1):87-108. 